\title{
Output Feedback Stabilization for Dynamic MIMO Semi-linear Stochastic Systems with Output Randomness Attenuation
}

\author{
Qichun Zhang \\ School of Engineering and \\ Sustainable Development \\ De Montfort University \\ Leicester, LE1 9BH, UK \\ Email: qichun.zhang@dmu.ac.uk
}

\author{
Liang $\mathrm{Hu}$ \\ School of Computer Science \\ and Informatics \\ De Montfort University \\ Leicester, LE1 9BH, UK \\ Email: liang.hu@dmu.ac.uk
}

\author{
John Gow \\ School of Engineering and \\ Sustainable Development \\ De Montfort University \\ Leicester, LE1 9BH, UK \\ Email: jgow@dmu.ac.uk
}

\begin{abstract}
In this paper, the problem of randomness attenuation is investigated for a class of MIMO semi-linear stochastic systems. To achieve this control objective, a $m$-block backstepping controller is designed to stabilize the closed-loop systems in probability sense. In addition, the output randomness attenuation can be achieved by optimising the design parameters using minimum entropy criterion. The effectiveness of this presented control algorithm can be verified by a given numerical example. In summary, the main contributions of this paper are characterized as follows: (1) an output feedback design method is adapted to stabilise the dynamic multi-variable semi-linear stochastic systems by block backstepping; (2) randomness of the system output is attenuated by searching the optimal design parameter based on the entropy criterion; (3) a framework of performance enhancement for stochastic systems is developed.
\end{abstract}

\section{INTRODUCTION}

Backstepping design method [1] has been presented as an effective approach for the controller design of SISO deterministic nonlinear control systems. For MIMO deterministic non-linear systems, [2][3] presented various controllers based on backstepping design. Recently, block backstepping design is investigated by Yaote Chang[4] and the extension of this results has been given in [5]. For stochastic systems, most of the results focus on the SISO systems[6]. However, very few results exist for the MIMO stochastic systems with block backstepping design.

For MIMO stochastic systems, the randomness of steady outputs can be bounded once the stochastic systems are stabilized. However, the randomness of the systems outputs would affect the control performance subjected to the random noises and couplings among the stochastic outputs[7]. Therefore, the investigation of the randomness is significant to enhance the performance of the MIMO stochastic systems. Notice that the probability density functions of these outputs obey nonGaussian distributions; hence the analysis using variance and covariance are not suitable to this problem. In this case, the entropy criterion is introduced to characterise the randomness of the system outputs. In other words, the purpose of this paper is to stabilize the stochastic systems and minimize the entropy of the system outputs due to the fact that there is no existing solution to attenuate the randomness of a MIMO Semi-linear stochastic system.

Motivated by the block backstepping design, the output feedback stabilization for a class of MIMO semi-linear stochastic systems are investigated in the paper due to the fact that the semi-linear stochastic systems become a significant research topic[8]. Based upon the system model and the control objective, a novel observer-based output feedback controller is design by $m$-block backstepping which can be used to stabilized the MIMO stochastic systems. Furthermore, the controller design parameters can be optimised by minimizing the entropy criteria, where the entropy of the system outputs can be estimated by multidimensional kernel density estimation. Following this presented control algorithm, the stability of the closed-loop systems can be guaranteed in probability sense while the randomness of the system outputs are attenuated by the parametric optimisation.

\section{Preliminaries}

\section{A. Problem Description}

Consider the following MIMO semi-linear stochastic systems with $m$ blocks which can be formulated as follows:

$$
\begin{aligned}
d \bar{x}_{i} & =\left(A_{i} \bar{x}_{i}+\bar{x}_{i+1}\right) d t+G_{1}\left(\bar{x}_{1}\right) d \beta_{t}, i=1, \cdots, m-1 \\
d \bar{x}_{m} & =\left(A_{m} \bar{x}_{m}+\bar{u}\right) d t+G_{m}\left(\bar{x}_{1}\right) d \beta_{t} \\
\bar{y} & =\bar{x}_{1}
\end{aligned}
$$

where $\beta_{t}$ is the s-dimensional vector-valued Wiener process, $\bar{x}_{i}$ is the $n$-dimensional state vector for $i$-th block, $A_{i}$ stands for the coefficient matrices with appropriate dimension, $G_{i}(\cdot)$ are $n$-dimensional nonlinear function. $\bar{y}$ and $\bar{u}$ are the system output vector and the vector-valued control input, respectively. The underlying probability space is triple $(\Omega, \mathscr{F}, \mathrm{P})$, where $\Omega$ is the sample space of continuous functions, $\mathscr{F}$ is a filtration adapted to the Wiener process $\beta_{t}$, and $\mathrm{P}$ is the reference probability measure on $\Omega$ 
Note that the system described above is in block strictfeedback format where the system outputs and control inputs are with the same dimension. As mentioned in Section I, the control objective is to stabilize this stochastic system in probability sense and minimize the entropy of the stochastic outputs. Before presenting the control algorithm, an assumption about the nonlinear function $G_{i}(\cdot)$ is given as follows:

Assumption 1: The $n \times s$ nonlinear function $G_{i}(\cdot)$ for each block of the semi-linear stochastic systems(1) satisfies

$$
\left\|G_{i}\left(X_{i}\right)\right\|_{2} \leq \sigma_{i}
$$

where $\|\cdot\|_{2}$ denotes the induced Euclidean norm for matrices and $\sigma_{i}$ is one positive real constant.

\section{B. Stability in Probability Sense}

Consider the general stochastic nonlinear system:

$$
d x=f(x) d t+g(x) d w
$$

where $x \in \mathbb{R}^{n}$ is the state, $w$ is an $r$-dimensional independent standard Wiener process, the underlying probability space is the triple $(\Omega, \mathscr{F}, \mathrm{P})$, and $f: \mathbb{R}^{n} \rightarrow \mathbb{R}^{n}$ and $g: \mathbb{R}^{n} \rightarrow \mathbb{R}^{n \times r}$ are locally Lipschitzian and with the following initial values

$$
f(0)=0, g(0)=0
$$

Definition 1 ([9]): The solution process $\{x(t), t \geq 0\}$ of the stochastic system (3) is said to be bounded in probability if $\lim _{t \rightarrow \infty} \sup _{0 \leq c \leq \infty} P\{|x(t)|>c\}=0$

Definition 2 ([10]): For any given $V(x) \in \mathscr{C}^{1,2}$, associated with the stochastic differential equation (3), the differential operator $\mathscr{L}$ can be defined as follows:

$$
\mathscr{L} V=\frac{\partial V}{\partial x} f(x)+\frac{1}{2} \operatorname{Tr}\left\{g^{T}(x) \frac{\partial^{2} V}{\partial x^{2}} g(x)\right\}
$$

We recall the following lemma [6] which gives the sufficient conditions on the boundedness in probability sense.

Lemma 1 ([6]): Consider system (3) and suppose that there exists a positive-define and radially unbounded function $V(x) \in \mathscr{C}^{1,2}, \mu_{1}(\cdot), \mu_{2}(\cdot) \in \mathscr{K}_{\infty}$, positive-define and radially unbounded function $W(x)$ and constant $c>0$ such that

$$
\begin{aligned}
& \mu_{1}(|x|) \leq V(x) \leq \mu_{2}(|x|) \\
& \mathscr{L} V(x) \leq-W(x)+c
\end{aligned}
$$

then the solution process of the system (3) is bounded in probability sense.

\section{Entropy and Kernel Density Estimation}

The information theory has been introduced by [11], where entropy can be used as a measure of the uncertainty of the random variables. For various purposes, a lot of different definitions of the entropy have been presented such as Shannon entropy, Min-entropy, Hartley entropy and Rényi's entropy. In this paper, the quadratic Rényi's entropy is selected to use, which has been introduced by [12] with the form

$$
H_{2}(\bar{y})=-\log \int \gamma^{2}(\bar{y}) d \bar{y}
$$

where $\gamma(\cdot)$ stands for the joint probability density functions (JPDF) of the system outputs. The data-based multidimensional kernel density estimation (MKDE) [13] is used to estimate the JPDF of the random variables. Note that entropy is equivalent to variance for Gaussian variable [14].

For vector-valued continuous system outputs $\bar{y} \in \mathbb{R}^{n}$, with its sampled data points $\left\{\bar{y}_{k}: k=1, \ldots, N\right\}$, the probability density function system output $\bar{y}$ can be estimated as follows:

$$
\hat{\gamma}(\bar{y})=\frac{1}{N} \sum_{k=1}^{N} G_{\Sigma}\left(\bar{y}-\bar{y}_{k}\right)
$$

where $G_{\Sigma}(\cdot)$ is the Gaussian function defined as follows:

$$
G_{\Sigma}(x)=(2 \pi)^{-\frac{n}{2}}(\operatorname{det} \Sigma)^{-\frac{1}{2}} \exp \left(-\frac{1}{2} x^{T} \Sigma^{-1} x\right)
$$

Since the JPDF can be estimated by MKDE, Eq.(7) can be rewritten as follows:

$$
H_{2}(\bar{y})=-\log V(\bar{y})
$$

where $V(\cdot)$ stands for information potential [11]. Furthermore, it can be approximated by

$$
\hat{V}(\bar{y})=\frac{1}{N^{2}} \sum_{i, j=1}^{N} G_{\sqrt{2} \Sigma}\left(\bar{y}_{i}-\bar{y}_{j}\right)
$$

\section{Control Algorithm}

\section{A. Linear estimator design}

The estimator can be designed as follows.

$$
\begin{aligned}
d \hat{\bar{x}}_{i} & =\left(A_{i} \hat{\bar{x}}_{i}+\hat{\bar{x}}_{i+1}+L_{i}\left(\bar{y}-\hat{\bar{x}}_{1}\right)\right) d t, i=1, \cdots, m-1 \\
d \hat{\bar{x}}_{m} & =\left(A_{m} \hat{\bar{x}}_{m}+\bar{u}+L_{m}\left(\bar{y}-\hat{\bar{x}}_{1}\right)\right) d t
\end{aligned}
$$

where $L_{i}$ is the gain of estimator.

The error of the estimator can be introduced as $\tilde{\bar{x}}=\bar{x}-\hat{\bar{x}}$ which satisfies

$$
\begin{aligned}
d \tilde{\bar{x}} & =\left[\begin{array}{cccc}
A_{1}-L_{1} & I & & \\
-L_{2} & A_{2} & \ddots & \\
\vdots & \vdots & \ddots & I \\
-L_{m} & 0 & \ldots & A_{m}
\end{array}\right] \tilde{\bar{x}} d t+\left[\begin{array}{c}
G_{1}(\bar{y}) \\
G_{2}(\bar{y}) \\
\vdots \\
G_{m}(\bar{y})
\end{array}\right] d \beta_{t} \\
& =A_{0} \tilde{\bar{x}} d t+G_{0}(\bar{y}) d \beta_{t}
\end{aligned}
$$

Using the linear observer design method, $A_{0}$ should be designed to be Hurwitz, then the semi-linear stochastic system with estimator can be re-expressed as follows:

$$
\begin{aligned}
d \tilde{\bar{x}} & =A_{0} \tilde{\bar{x}} d t+G_{0}(\bar{y}) d \beta_{t} \\
d \bar{x}_{1} & =\left(A_{1} \bar{x}_{1}+\tilde{\bar{x}}_{2}+\hat{\bar{x}}_{2}\right) d t+G_{1}(\bar{y}) d \beta_{t} \\
d \hat{\bar{x}}_{i} & =\left(F_{i}+\hat{\bar{x}}_{i+1}\right) d t, i=2, \cdots, m-1 \\
d \hat{\bar{x}}_{m} & =\left(F_{m}+\bar{u}\right) d t \\
\bar{y} & =\bar{x}_{1}
\end{aligned}
$$

where $F_{i}=A_{i} \hat{\bar{x}}_{i}+L_{i}\left(\bar{y}-\hat{\bar{x}}_{1}\right), i=2, \cdots, m$ 


\section{B. Block backstepping controller design}

Since the semi-linear stochastic system with linear estimator is in the strict feedback form, block backstepping can be applied for this MIMO case.

For each block of the original system(1), consider $\bar{\varphi}_{i}\left(\bar{y}, \hat{x}_{i}\right)$ as the virtual input which can be rewritten as

$$
\bar{\varphi}_{i}\left(\bar{y}, \hat{x}_{i}\right)=\left[\varphi_{i 1}\left(\bar{y}, \hat{x}_{i}\right), \ldots, \varphi_{i n}\left(\bar{y}, \hat{x}_{i}\right)\right]^{T}
$$

where $\hat{x}_{i}=\left[\bar{x}_{1}, \hat{\bar{x}}_{2}, \ldots, \hat{\bar{x}}_{i}\right], i=1, \cdots, m-1$.

Then the vector-valued error variables can be presented by

$$
\bar{z}_{i}=\hat{\bar{x}}_{i+1}-\bar{\varphi}_{i}\left(\bar{y}, \hat{x}_{i}\right)
$$

where $\bar{z}_{i}=\left[z_{i 1}, \ldots, z_{i n}\right]^{T}, i=1, \cdots, m-1$.

Using Ito's lemma, we have

$$
\begin{aligned}
d \bar{z}_{i} & =\left[\left(F_{i+1}+\hat{\bar{x}}_{i+2}\right)-\Phi_{1}\left(F_{1}+\hat{\bar{x}}_{2}\right)-\frac{1}{2} \Pi_{1}\right. \\
& \left.-\sum_{l=2}^{i} \Phi_{l}\left(F_{l}+\hat{\bar{x}}_{l+1}\right)\right] d t-\Phi_{1} G_{1}(\bar{y}) d \beta_{t} \\
& =\left(\Xi_{i}+\hat{\bar{x}}_{i+2}\right) d t-\Phi_{1} G_{1}(\bar{y}) d \beta_{t}
\end{aligned}
$$

where

$$
\begin{aligned}
& \Phi_{1}=\left[\nabla_{\bar{y}} \varphi_{11}(\bar{y}), \ldots, \nabla_{\bar{y}} \varphi_{1 n}(\bar{y})\right]^{T} \\
& \Phi_{l}= {\left[\nabla_{\hat{\bar{x}}_{l}} \varphi_{l 1}\left(\bar{y}, \hat{x}_{l}\right), \ldots, \nabla_{\hat{x}_{l}} \varphi_{l n}\left(\bar{y}, \hat{x}_{l}\right)\right]^{T} } \\
& \Pi_{1}=\left[\begin{array}{l}
\operatorname{Tr}\left\{G_{1}^{T}(\bar{x})\left(H_{\bar{x}} \varphi_{11}\left(\bar{x}_{1}\right)\right) G_{1}\left(\bar{x}_{1}\right)\right\} \\
\vdots \\
\operatorname{Tr}\left\{G_{1}^{T}(\bar{x})\left(H_{\bar{x}} \varphi_{1 n}\left(\bar{x}_{1}\right)\right) G_{1}\left(\bar{x}_{1}\right)\right\}
\end{array}\right] \\
& \Xi_{i}=F_{i+1}-\Phi_{1}\left(F_{1}+\hat{\bar{x}}_{2}\right)-\frac{1}{2} \Pi_{1}-\sum_{l=2}^{i} \Phi_{l}\left(F_{l}+\hat{\bar{x}}_{l+1}\right)
\end{aligned}
$$

To stabilize the entire stochastic system, we employ a Lyapunov function candidate which is presented as follows:

$$
V=\frac{1}{2} \sum_{k=1}^{n} \bar{y}_{k}^{2}+\frac{b}{2}\left(\tilde{x}^{T} P \tilde{x}\right)^{2}+\frac{1}{4} \sum_{i=1}^{m-1} \sum_{l=1}^{n} \bar{z}_{i l}^{4}
$$

where $P$ denotes the positive definite matrix which satisfies $A_{0}{ }^{T} P+P A_{0}<0$.

Now we start to analyze the property of $\mathscr{L} V$ for the proposed Lypunov function candidate.

$$
\begin{aligned}
\mathscr{L} V & =\bar{y}^{T}\left(A_{1} \bar{y}+\tilde{\bar{x}}_{2}+\hat{\bar{x}}_{2}\right)+\frac{1}{2} \operatorname{Tr}\left\{G_{1}^{T}(\bar{y}) G_{1}(\bar{y})\right\} \\
& +\sum_{i=1}^{m-1} \eta_{i}\left(\Xi_{i}+\hat{\bar{x}}_{i+2}\right)+\frac{3}{2} \operatorname{Tr}\left\{\Gamma_{i} \Phi_{1} G_{1}(\bar{y})\left(\Phi_{1} G_{1}(\bar{y})\right)^{T}\right\} \\
& +2 b \operatorname{Tr}\left\{G_{0}^{T}(\bar{y})\left(2 P \tilde{x} \tilde{x}^{T} P+\tilde{x}^{T} P \tilde{x} P\right) G_{0}(\bar{y})\right\} \\
& -b \tilde{x}^{T} P \tilde{x}\|\tilde{x}\|^{2}
\end{aligned}
$$

where

$$
\begin{aligned}
\eta_{i} & =\left[z_{i 1}^{3}, \ldots, z_{i n}^{3}\right] \\
\Gamma_{i} & =\operatorname{diag}\left(z_{i 1}^{2}, \ldots, z_{i n}^{2}\right)
\end{aligned}
$$

The trace terms of $\mathscr{L} V$ remain difficult to handle, thus a useful lemma is given here which can be used repeatedly to simplify the formulation.

Lemma 2: Consider that $A_{1}, A_{2}, B \in R^{n \times n}$ are $n$ dimensional square matrices and $D \in R^{n \times n}$ is diagonal matrix, where $A_{1}=\left[\bar{a}_{11}, \ldots, \bar{a}_{1 n}\right]^{T}, A_{2}=\left[\bar{a}_{21}, \ldots, \bar{a}_{2 n}\right]$ and $D=\operatorname{diag}\left\{d_{1}, \ldots, d_{n}\right\}$. Then the following inequality holds.

$$
\operatorname{Tr}\left\{D A_{1} B A_{2}\right\} \leq \sum_{i=1}^{n}\left\|d_{i}\right\|\left\|\bar{a}_{1 i}\right\|\left\|\bar{a}_{2 i}\right\|\|B\|
$$

Proof: Using the structure of the matrices which has been mentioned above, we can have

$$
\begin{aligned}
& \operatorname{Tr}\left\{D A_{1} B A_{2}\right\} \\
& =\operatorname{Tr}\left\{\left[\begin{array}{lll}
d_{1} & & \\
& \ddots & \\
& & d_{n}
\end{array}\right]\left[\begin{array}{c}
a_{11} \\
\vdots \\
a_{1 n}
\end{array}\right] B\left[\begin{array}{ccc}
a_{21} & \cdots & a_{2 n}
\end{array}\right]\right\} \\
& =\operatorname{Tr}\left\{\left[\begin{array}{llll}
d_{1} & & \\
& \ddots & \\
& & d_{n}
\end{array}\right]\left[\begin{array}{ccc}
a_{11} B a_{21} & \ldots & a_{11} B a_{2 n} \\
\vdots & \ddots & \vdots \\
a_{1 n} B a_{21} & \ldots & a_{1 n} B a_{2 n}
\end{array}\right]\right\} \\
& =\sum_{i=1}^{n} d_{i} a_{1 i} B a_{2 i} \\
& \leq \sum_{i=1}^{n}\left\|d_{i} a_{1 i} B a_{2 i}\right\|
\end{aligned}
$$

Following the property of the norm operation, we have

$$
\sum_{i=1}^{n}\left\|d_{i} a_{1 i} B a_{2 i}\right\| \leq \sum_{i=1}^{n}\left\|d_{i}\right\|\left\|a_{1 i}\right\|\left\|a_{2 i}\right\|\|B\|
$$

which ends the proof of Lemma 2

Based upon Lemma 2, we can obtain the following inequalities in order to deal with the trace terms in Eq.(20) separately using Young's inequality.

At firstly, we have

$$
\begin{aligned}
\operatorname{Tr} & \left\{\Gamma \Phi_{1} G_{1}(\bar{y}) G_{1}^{T}(\bar{y}) \Phi_{1}^{T}\right\} \\
& \leq \sum_{i=1}^{n} z_{1 i}^{2}\left\|\nabla_{\bar{x}} \varphi_{1 i}^{T}(\bar{y})\right\|_{2}\left\|\nabla_{\bar{x}} \varphi_{1 i}(\bar{y})\right\|_{2}\left\|G_{1}(\bar{y})\right\|_{2}\left\|G_{1}^{T}(\bar{y})\right\|_{2} \\
& \leq \sum_{i=1}^{n} \frac{\varepsilon_{1 i}^{2}}{2} z_{1 i}^{4}\left\|\nabla_{\bar{x}} \varphi_{1 i}^{T}(\bar{y})\right\|^{2}+\sum_{i=1}^{n} \frac{1}{2 \varepsilon_{1 i}^{2}}\left\|G_{1}(\bar{y})\right\|_{2}^{4} \\
& =\sum_{i=1}^{n} \frac{\varepsilon_{1 i}^{2}}{2} z_{1 i}^{4}\left\|\nabla_{\bar{x}} \varphi_{1 i}^{T}\left(\bar{x}_{1}\right)\right\|^{2}+\sum_{i=1}^{n} \frac{1}{2 \varepsilon_{1 i}^{2}} \sigma_{1}^{4}
\end{aligned}
$$

Next, we further obtain

$$
\operatorname{Tr}\left\{G_{1}^{T}(\bar{y}) G_{1}(\bar{y})\right\} \leq n\left\|G_{1}(\bar{y})\right\|^{2}=n \sigma_{1}^{2}
$$


and

$$
\begin{aligned}
2 b T r & \left\{G_{0}^{T}(\bar{y})\left(2 P \tilde{x} \tilde{x}^{T} P+\tilde{x}^{T} P \tilde{x} P\right) G_{0}(\bar{y})\right\} \\
& =4 b\left\|G_{0}^{T}(\bar{y}) P \tilde{x}\right\|_{F}^{2}+2 b \tilde{x}^{T} P \tilde{x} T r\left\{P G_{0}(\bar{y}) G_{0}^{T}(\bar{y})\right\} \\
& \leq 4 b \sqrt{s}\left\|G_{0}^{T}(\bar{y})\right\|^{2}\|P\|^{2}\|\tilde{x}\|^{2} \\
& +2 b\left\|\operatorname{Tr}\left\{P G_{0}(\bar{y}) G_{0}^{T}(\bar{y})\right\}\right\|\|P\|\|\tilde{x}\|^{2} \\
& \leq\left(\frac{\tilde{\varepsilon}_{1}^{2}+\tilde{\varepsilon}_{2}^{2}}{2}\right)\|\tilde{x}\|^{4}+\frac{8 b^{2} s}{\tilde{\varepsilon}_{1}^{2}}\left\|G_{0}^{T}(\bar{y})\right\|^{4}\|P\|^{4} \\
& +\frac{2 b^{2}}{\tilde{\varepsilon}_{2}^{2}}\|P\|^{2}\left(\sum_{i=1}^{n m}\left|p_{i}\right|\left\|G_{0}^{T}(\bar{y})\right\|^{2}\right)^{2} \\
& =\left(\frac{\tilde{\varepsilon}_{1}^{2}+\tilde{\varepsilon}_{2}^{2}}{2}\right)\|\tilde{x}\|^{4}+\tilde{c}
\end{aligned}
$$

where $\tilde{\varepsilon}_{1}, \tilde{\varepsilon}_{2}, \varepsilon_{1 i}$ are any real positive numbers. $\|\cdot\|_{F}$ stands for Frobenius norm.

Moreover, notice that $\tilde{c} \geq 0$,

$\tilde{c}=\frac{8 b^{2} s}{\tilde{\varepsilon}_{1}^{2}}\left\|G_{0}^{T}(\bar{y})\right\|^{4}\|P\|^{4}+\frac{2 b^{2}}{\tilde{\varepsilon}_{2}^{2}}\|P\|^{2}\left(\sum_{i=1}^{n m} \mid p_{i}\left\|G_{0}^{T}(\bar{y})\right\|^{2}\right)^{2}$

and the following inequality always holds

$$
-b \tilde{x}^{T} P \tilde{x}\|\tilde{x}\|^{2} \leq-b \lambda_{\min }\{P\}\|\tilde{x}\|^{4}
$$

Substituting these inequalities Eq. (23)-Eq. (26) to $\mathscr{L} V$, as a result, Eq. (20) can be rewritten as the following inequality which can be used to evaluate the controller for stabilization.

$$
\begin{aligned}
& \mathscr{L} V \leq \bar{y}^{T}\left(A_{1} \bar{y}+\tilde{\bar{x}}_{2}+\hat{\bar{x}}_{2}\right)+\frac{1}{2} n \sigma_{1}^{2}+\sum_{i=1}^{m-1} \eta_{i}\left(\Xi_{i}+\hat{\bar{x}}_{i+2}\right) \\
& +\frac{3}{2}\left(\sum_{i=1}^{n} \frac{\varepsilon_{1 i}^{2}}{2} z_{1 i}^{4}\left\|\nabla_{\bar{x}} \varphi_{1 i}^{T}\left(\bar{x}_{1}\right)\right\|^{2}+\sum_{i=1}^{n} \frac{1}{2 \varepsilon_{1 i}^{2}} \sigma_{1}^{4}\right) \\
& -b \lambda_{\min }\{P\}\|\tilde{x}\|^{4}+\left(\frac{\tilde{\varepsilon}_{1}^{2}+\tilde{\varepsilon}_{2}^{2}}{2}\right)\|\tilde{x}\|^{4}+\tilde{c} \\
& =\bar{y}^{T}\left(A_{1} \bar{y}+\tilde{\bar{x}}_{2}+\hat{\bar{x}}_{2}\right) \\
& +\sum_{i=2}^{m-1} \eta_{i}\left(\Xi_{i}+\bar{z}_{i+1}+\bar{\varphi}_{i+1}\left(\bar{y}, \hat{x}_{i+1}\right)\right) \\
& +\eta_{1}\left(\Xi_{1}+\bar{z}_{2}+\bar{\varphi}_{2}\left(\bar{y}, \hat{\bar{x}}{ }_{2}\right)+\left[\begin{array}{c}
\frac{\varepsilon_{11}^{2}}{2} z_{11}\left\|\nabla_{\bar{x}} \varphi_{11}^{T}(\bar{y})\right\|^{2} \\
\vdots \\
\frac{\varepsilon_{1 n}^{2}}{2} z_{1 n}\left\|\nabla_{\bar{x}} \varphi_{1 n}^{T}(\bar{y})\right\|^{2}
\end{array}\right]\right) \\
& +\frac{3}{2} \sum_{i=1}^{n} \frac{1}{2 \varepsilon_{1 i}^{2}} \sigma_{1}^{4}+\tilde{c}-\left(b \lambda_{\min }\{P\}-\frac{\tilde{\varepsilon}_{1}^{2}+\tilde{\varepsilon}_{2}^{2}}{2}\right)\|\tilde{x}\|^{4}
\end{aligned}
$$

Based upon Lemma 1, the control input and virtual inputs can be chosen as

$$
\begin{aligned}
\bar{\varphi}_{1}(\bar{y}) & =\left(-W-A_{1}\right) \bar{y}-\tilde{\bar{x}}_{2} \\
\bar{\varphi}_{2}\left(\bar{y}, \hat{\bar{x}}_{2}\right) & =-\Xi_{1}-\bar{z}_{2}-\Theta-C_{1} \bar{z}_{1} \\
\bar{\varphi}_{i+1}\left(\bar{y}, \hat{x}_{i+1}\right) & =-\Xi_{i}-\bar{z}_{i+1}-C_{i} \bar{z}_{i} \\
u=\bar{\varphi}_{m}\left(\bar{y}, \hat{x}_{m}\right) & =-\Xi_{m-1}-\bar{z}_{m}-C_{m-1} \bar{z}_{m-1}
\end{aligned}
$$

where $W$ denotes the positive definite matrix.

$$
\begin{gathered}
\Theta=\left[\begin{array}{c}
\frac{\varepsilon_{11}^{2}}{2} z_{11}\left\|\nabla_{\bar{x}} \varphi_{11}^{T}(\bar{y})\right\|^{2} \\
\vdots \\
\frac{\varepsilon_{1 n}^{2}}{2} z_{1 n}\left\|\nabla_{\bar{x}} \varphi_{1 n}^{T}(\bar{y})\right\|^{2}
\end{array}\right] \\
C_{i}=\operatorname{diag}\left[c_{i 1}, \ldots, c_{i n}\right], c_{i j}>0
\end{gathered}
$$

Furthermore, $\mathscr{L} V$ can be rewritten as

$$
\mathscr{L} V=-\bar{y}^{T} W \bar{y}-\tilde{p}\|\tilde{x}\|^{4}-\sum_{i=1}^{m-1} \sum_{l=1}^{n} c_{i l} \bar{z}_{i l}^{4}+\bar{c}
$$

where

$$
\tilde{p}=b \lambda_{\min }\{P\}-\frac{\tilde{\varepsilon}_{1}^{2}+\tilde{\varepsilon}_{2}^{2}}{2}, \bar{c}=\frac{3}{2} \sum_{i=1}^{n} \frac{1}{2 \varepsilon_{1 i}^{2}} \sigma_{1}^{4}+\tilde{c}
$$

Thus, the stabilisation of the closed-loop semi-linear stochastic system is analysed by the following theorem in which the structure of the controller that can stabilise the presented closed-loop stochastic system.

Theorem 1: The semi-linear stochastic system (1) with linear estimator (12) and control law (31) are guaranteed to be bounded in probability sense if there exists a positive definite matrix $P$ which makes $\tilde{p}>0$ and $A_{0}{ }^{T} P+P A_{0}<0$.

Proof: Since $A_{0}, P$ and $\tilde{p}$ are defined in Equations $(13),(19)$ and (34), the proof is showed above.

\section{Output Randomness Optimization}

As mentioned in Section II, the performance criterion can be given as follows:

$$
J_{k}=-\log \hat{V}\left(\bar{y}, W_{0}\right)
$$

where $W_{0}$ denotes the set of design parameters, $\mathrm{W}_{0}=$ $\left\{W, \tilde{\varepsilon}_{1}, \tilde{\varepsilon}_{2}, \varepsilon_{1 i}, C_{i}\right\}, i=1, \ldots, n . k$ stands for the sampling instant. Since the $\log (\cdot)$ function is with the monotonic increasing property, minimizing of the entropy is equivalent to maximizing the information potential, thus the performance criterion can be further simplified only using $\hat{V}\left(\bar{y}, W_{0}\right)$.

In addition, the following theorem is given to claim that this performance criterion is a globally convex function based on another assumption.

Assumption 2: The closed-loop stochastic system output vector $\bar{y}$ satisfies the following inequality:

$$
\frac{\partial \bar{y}}{\partial W_{0}} \leq M
$$

where the real positive matrix $M$ denotes the upper bound.

Theorem 2: For the presneted control algorithm, there exists a real positive number $\delta_{0}>0$, such that the information potential is globally concave with respect to the design parameter $W_{0}$ for all $\lambda_{\min }(\Sigma)>\delta_{0}$. Thus the equivalent performance criterion (35) is convex with a global optimum. 
Proof: Denote $\varepsilon_{i j, k}=\bar{y}_{i, k}-\bar{y}_{j, k}$, then we have

$$
\begin{aligned}
& \frac{\partial^{2} \hat{V}_{k}\left(W_{0}\right)}{\partial W_{0}^{2}}=\frac{1}{N^{2}} \frac{\partial}{\partial W_{0}} \sum_{i, j=1}^{N} \frac{\partial}{\partial W_{0}} G_{\sqrt{2} \Sigma}\left(\varepsilon_{i j, k}\right) \\
& =\frac{1}{N^{2}} \frac{\partial}{\partial W_{0}} \sum_{i, j=1}^{N} \frac{\partial G_{\sqrt{2} \Sigma}\left(\varepsilon_{i j, k}\right)}{\partial \varepsilon_{i j, k}} \frac{\partial \varepsilon_{i j, k}}{\partial W_{0}} \\
& \leq-\frac{1}{N^{2}}(\sqrt{2} \Sigma)^{-1} \frac{\partial}{\partial W_{0}} \sum_{i, j=1}^{N} G_{\sqrt{2} \Sigma}\left(\varepsilon_{i j, k}\right) \times \varepsilon_{i j, k} M \\
& =-\frac{M}{N^{2}}(\sqrt{2} \Sigma)^{-1} \sum_{i, j=1}^{N} G_{\sqrt{2} \Sigma}\left(\varepsilon_{i j, k}\right) \\
& \times\left(\varepsilon_{i j, k}^{T}\left(M-(\sqrt{2} \Sigma)^{-1}\right) \varepsilon_{i j, k}\right)
\end{aligned}
$$

As a result, $\frac{\partial^{2} V_{k}(\varepsilon)}{\partial W_{0}^{2}} \leq 0$ if $M \geq(\sqrt{2} \Sigma)^{-1}$. It is shown that the eigenvalues of $\frac{\partial^{2} V_{k}(\varepsilon)}{\partial W_{0}^{2}}$ approach $0^{-}$as $\lambda_{\min }(\Sigma)$ goes to infinity. Based on the Lemma 3 in [15], $\hat{V}_{k}\left(\bar{y}, W_{0}\right)$ will be concave since $\lambda_{\min }(\Sigma)$ is sufficiently large. Moreover, the performance criterion (35) is convex which results in the global optimum. It shows that the proof is completed.

Since the performance criterion is convex then the standard convex optimization approach can be applied to this issue directly. Without loss of generality, the gradient descent optimization is given as follows:

$$
W_{0, k+1}=W_{0, k}-\left.\gamma \frac{\partial \hat{V}_{k}\left(W_{0}\right)}{\partial W_{0}}\right|_{W_{0}=W_{0, k}}
$$

where $\gamma>0$ denotes the pre-specified step.

\section{AN NUMERICAL EXAMPLE}

To demonstrate the presented algorithm procedure, a multivariable semi-linear stochastic system is shown as follows:

$$
\begin{aligned}
d \bar{x}_{1} & =\left(\left[\begin{array}{cc}
-1 & 0.5 \\
0 & -2
\end{array}\right] \bar{x}_{1}+\bar{x}_{2}\right) d t+\sin \left(\bar{x}_{1}\right) d \beta_{t} \\
d \bar{x}_{2} & =\left(\left[\begin{array}{cc}
-1.5 & 0 \\
-0.5 & -1
\end{array}\right] \bar{x}_{2}+\bar{u}\right) d t+\cos \left(\bar{x}_{1}\right) d \beta_{t} \\
\bar{y} & =\bar{x}_{1}
\end{aligned}
$$

where $A_{1}, A_{2}, G_{1}$ and $G_{2}$ are given as coefficient matrices.

In addition, the linear estimator can be obtained with the feedback gain matrices $L_{1}=L_{2}=\operatorname{diag}\{15,15\}$. Thus, the closed-loop system with estimator can be rewritten by

$$
\begin{aligned}
& d \bar{y}=\left(\left[\begin{array}{cc}
-1 & 0.5 \\
0 & -2
\end{array}\right] \bar{y}+\tilde{\bar{x}}_{2}+\hat{\bar{x}}_{2}\right) d t+\sin (\bar{y}) d \beta_{t} \\
& d \hat{\bar{x}}_{2}=\left(\left[\begin{array}{cc}
-1.5 & 0 \\
-0.5 & -1
\end{array}\right] \hat{\bar{x}}_{2}+\left[\begin{array}{cc}
2 & 0 \\
0 & 3
\end{array}\right] \tilde{\bar{x}}_{1}+\bar{u}\right) d t \\
& d\left[\begin{array}{c}
\tilde{\bar{x}}_{1} \\
\tilde{\bar{x}}_{2}
\end{array}\right]=\left[\begin{array}{cccc}
-3 & 0.5 & 1 & 0 \\
0 & -5 & 0 & 1 \\
-2 & 0 & -1.5 & 0 \\
0 & -3 & -0.5 & -1
\end{array}\right]\left[\begin{array}{c}
\tilde{\bar{x}}_{1} \\
\tilde{\bar{x}}_{2}
\end{array}\right] d t \\
&+\left[\begin{array}{c}
\sin (\bar{y}) \\
\cos (\bar{y})
\end{array}\right] d \beta_{t}
\end{aligned}
$$

where $A_{0}$ and $G_{0}$ are obtained with $A_{0}$ being Hurwitz.

As we mentioned in section III, the first virtual control input and the control input can be designed using Eq.(31). It has been shown that the design parameters $W_{0}$ will affect the performance of the controller, then the optimisation is essential while $W=\operatorname{diag}\{-20,25\}$ can be pre-selected and other parameters can be initialized by positive random numbers.

In this simulation, the sampling time $k$ is selected as $0.01 s$, and the control performance of the closed-loop stochastic system are given by curves in Figs. 1-3. The output trajectories are indicated by Fig.1. In particular, the stochastic outputs have been stabilized rapidly. The control input is depicted by Fig.2. In Fig.3, the performance criterion are given using $\mathrm{KDE}$ which results in a smooth curve. It shows that the value of $J$ descends when the presented optimisation method searches of the optimal design parameters. Simultaneously, the randomness of the system outputs has been minimised. Notice that $G_{2}(0) \neq 0$, thus the output trajectories are just bounded in probability sense. If we change the model as $G_{2}(\bar{y})=\sin (\bar{y})$, then the output will convergent to 0 in probability sense and the performance can be shown by Fig. 4

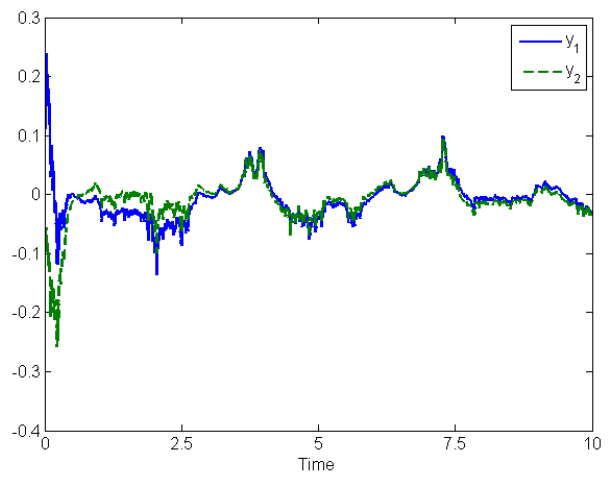

Fig. 1. Output trajectories of the closed-loop stochastic system

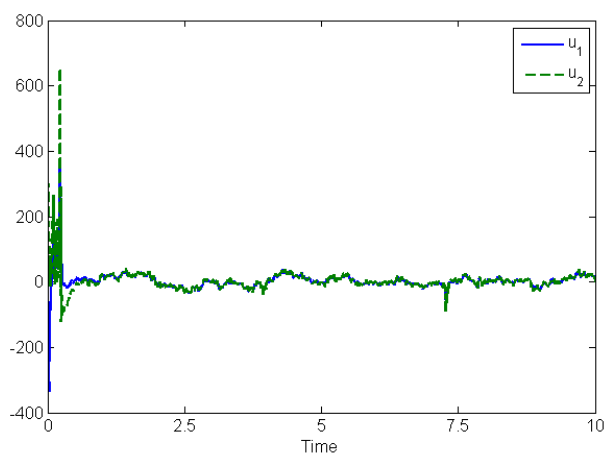

Fig. 2. The control input signal

\section{FURTHER DISCUSSION}

The presented control algorithm is very convenient to extend to bilinear stochastic systems [10] which implies that Assumption 1 in this paper can be released to the Lipschitz condition. 


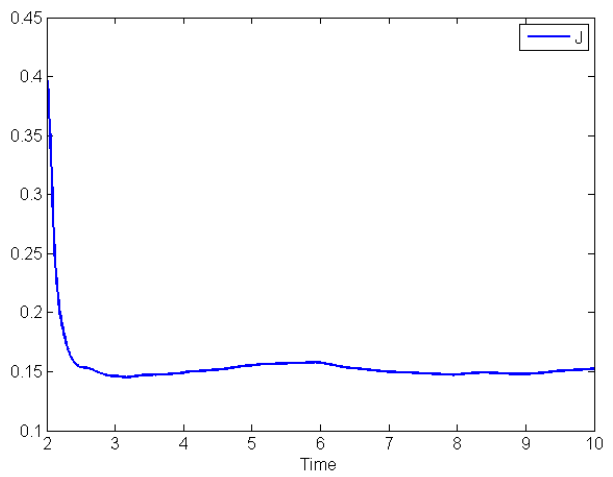

Fig. 3. The value of performance criteria $J$

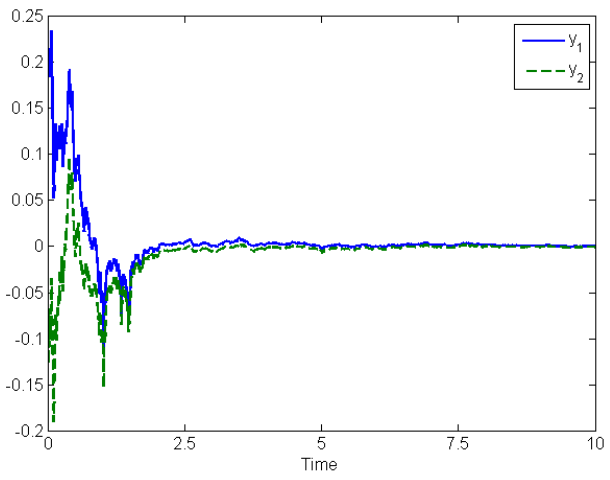

Fig. 4. Output trajectories of the control stochastic system with $G_{2}(0)=0$

Then the outputs of the stochastic systems can be stabilized using block backstepping design while the performance of the systems can be enhanced by optimizing the design parameters. Naturally, not only the randomness attenuation problem can be investigated but also other control objectives can be considered following various performance criteria. Therefore, we can extend this control algorithm as a framework of performance enhancement for a class of dynamic stochastic systems.

Particularly, the couplings among the system outputs can also be investigated using this presented framework. Based on the concept of probabilistic decoupling, the performance criterion can be described using probability density functions of the system outputs as follows.

$$
J_{k}=\min _{W_{0}}\left\|\gamma(\bar{y})-\gamma_{i}\left(y_{i}\right)\right\|
$$

where $\gamma_{i}(\cdot)$ stands for the probability density function (PDF) of each system outputs which can also be approximated by kernel density estimation (KDE).

\section{CONCLUSIONS}

For a class of MIMO semi-linear stochastic systems, a output feedback control algorithm is presented for the problem of transient randomness attenuation. Based on the linear estimator and the $m$-block backstepping design, the structure of the controller is obtained to stabilize the closed- loop stochastic system. Since the design parameters of the controller affect the performance of the system outputs, the minimum entropy performance criterion is given to attenuate the output randomness of the closed-loop system. Furthermore, the performance criterion can be restated using information potential equivalently. Based upon the multidimensional kernel density estimation, the convexity of the presented performance criteria can be guaranteed and standard convex optimization can be used to search the optimal parameters in this case. To verify the presented control algorithm, a numerical example is given while the simulation results show the effectiveness. In addition, this control algorithm can be considered as a framework because it can be extended to bilinear or even Lipschitz non-linear stochastic system and other performance criteria can also be considered by the similar approach, such as probabilistic decoupling, etc.

\section{ACKNOWLEDGEMENTS}

This work is partly supported by HEIF project 2018 at De Montfort University, this is acknowledged.

\section{REFERENCES}

[1] M. Krstic, I. Kanellakopoulos, and P. V. Kokotovic, Nonlinear and adaptive control design. Wiley, 1995.

[2] C. Wang and Y. Lin, "Multivariable adaptive backstepping control: a norm estimation approach," IEEE Transactions on Automatic Control, vol. 57, no. 4, pp. 989-995, 2012.

[3] S. S. Ge and Z. Li, "Robust adaptive control for a class of mimo nonlinear systems by state and output feedback," IEEE Transactions on Automatic Control, vol. 59, no. 6, pp. 1624-1629, 2014.

[4] Y. Chang, "Block backstepping control of mimo systems," IEEE Transactions on Automatic Control, vol. 56, no. 5, pp. 1191-1197, 2011.

[5] C. Cheng, G. Su, and C. Chien, "Block backstepping controllers design for a class of perturbed non-linear systems with m blocks," IET Control Theory \& Applications, vol. 6, no. 13, pp. 2021-2030, 2012.

[6] S.-J. Liu, J.-F. Zhang, and Z.-P. Jiang, "Decentralized adaptive outputfeedback stabilization for large-scale stochastic nonlinear systems," Automatica, vol. 43, no. 2, pp. 238-251, 2007.

[7] Q. Zhang, J. Zhou, H. Wang, and T. Chai, "Minimized coupling in probability sense for a class of multivariate dynamic stochastic control systems," in 2015 54th IEEE Conference on Decision and Control (CDC). IEEE, 2015, pp. 1846-1851.

[8] M. Yang and A. Armaou, "Feedback control of semi-linear distributed parameter systems using advanced pod method," in Decision and Control (CDC), 2015 IEEE 54th Annual Conference on. IEEE, 2015, pp. 46804687.

[9] R. Khasminskii, Stochastic stability of differential equations. Springer Science \& Business Media, 2011, vol. 66.

[10] Q. Zhang, J. Zhou, H. Wang, and T. Chai, "Output feedback stabilization for a class of multi-variable bilinear stochastic systems with stochastic coupling attenuation,' IEEE Transactions on Automatic Control, vol. 62, no. 6, pp. 2936-2942, 2017.

[11] T. M. Cover and J. A. Thomas, Elements of information theory. John Wiley \& Sons, 2012.

[12] Y. Zhou, Q. Zhang, H. Wang, P. Zhou, and T. Chai, "Ekf-based enhanced performance controller design for nonlinear stochastic systems," IEEE Transactions on Automatic Control, vol. 63, no. 4, pp. 1155-1162, 2018.

[13] Q. Zhang and A. Wang, "Decoupling control in statistical sense: minimised mutual information algorithm," International Journal of Advanced Mechatronic Systems, vol. 7, no. 2, pp. 61-70, 2016.

[14] Y. Zhou, Q. Zhang, and H. Wang, "Enhanced performance controller design for stochastic systems by adding extra state estimation onto the existing closed loop control," in Control (CONTROL), 2016 UKACC 11th International Conference on. IEEE, 2016, pp. 1-6.

[15] Y. Liu, H. Wang, and C. Hou, "Ukf based nonlinear filtering using minimum entropy criterion," IEEE Transactions on Signal Processing, vol. 61, no. 20, pp. 4988-4999, 2013. 